\title{
THE H/HE RATIO OF WN STARS
}

\author{
Paul A. Crowther, Linda J. Smith and Allan J. Willis \\ Department of Physics and Astronomy \\ University College London \\ Gower St, London WC1E 6BT \\ England
}

\begin{abstract}
The observed He II Pickering decrement is modelled using the escape probability method developed by Castor \& Van Blerkom (1970) to solve simultaneously the equations of radiative and statistical equilibrium for detailed model hydrogen and helium atoms. All important radiative and collisional processes are incorporated in this nLTE model. We confirm values of $0.0 \leq \mathrm{H} / \mathrm{He} \leq 0.5$ for WNE stars and $0.0 \leq \mathrm{H} / \mathrm{He} \leq 3.0$ for WNL stars with considerable spread in each subtype.
\end{abstract}

\section{Model Atom and Grids}

The model atom used contains 41 levels of $\mathrm{He}^{0}$ (every $S, P$, and $D$ state with $n \leq 8$ ), 30 levels of $\mathrm{He}^{+}$, the $\mathrm{He}^{2+}$ ion, plus 16 levels of $\mathrm{H}^{0}$ and the $\mathrm{H}^{+}$ion. Several model atoms were tested, including one with the same doubly-excited states incorporated by Bhatia and Underhill $(1986,1988)$. However, test calculations show that level populations are not significantly affected by these levels.

Since the detailed structure of WR stars remains uncertain, model results for a very large parameter space have been covered: $10^{9} \leq n_{\mathrm{e}} \leq 10^{12} \mathrm{~cm}^{-3}, 5 \times 10^{4} \leq T_{\mathrm{e}} \leq 10^{6} \mathrm{~K}$, $0.1 \leq \mathrm{H} / \mathrm{He} \leq 10.0$. The level populations were iterated until either 1 part in $10^{4}$ accuracy was achieved or 30 iterations performed. Those runs for which convergence was not achieved are expected to be accurate to within several percent. It is noted that when $T_{c}=T_{e}$, and the geometric dilution factor is set to unity, LTE populations are returned as expected.

Since the even quantum numbers of the He II Pickering $(n \rightarrow 4)$ series coincide with the $\mathrm{H}$ I Balmer lines $(n \rightarrow 2)$, the $\mathrm{H} / \mathrm{He}$ ratio is derived from the excess of the even ( $\mathrm{He} \mathrm{II}+\mathrm{H} \mathrm{I}$ ) over the odd (He II) lines in the series. Pickering decrements $\left(\log \Delta F_{\lambda}\right)$ were found from the mean of the logarithmic difference between the odd and even He II optically thin lines $(n=9-17)$.

\section{Results}

Model values of $\log \Delta F_{\lambda}$ were compared to those observed in Galactic and LMC WN stars, from Conti, Perry and Leep (1983). We find that for WNE stars $0.0 \leq \mathrm{H} / \mathrm{He} \leq 0.5$, and for WNL stars $0.0 \leq \mathrm{H} / \mathrm{He} \leq$ 3.0. Comparison of observed Pickering decrements with model grids are consistent over a wide range of $n_{\mathrm{e}}, T_{\mathrm{e}}, R_{\mathrm{c}}, T_{\mathrm{c}}$, and $\mathrm{H} / \mathrm{He}$, and confirm the hydrogen depletion of WN stars, and their chemically evolved nature.

Special thanks are given to Pete Storey for his help with the atomic rate calculations. 